\title{
EFFECTS OF IMPULSE FORCES ACTING ON THE EYE SOCKET WALLS - STRENGTH ANALYSIS
}

\author{
SEBASTIAN LiPA \\ Lodz University of Technology, Institute of Materials Science and Engineering, Lodz, Poland \\ Jerzy Mrozowski, Jan Awrejcewicz \\ Lodz University of Technology, Department of Automation, Biomechanics and Mechatronics, Lodz, Poland \\ e-mail: jan.awrejcewicz@p.lodz.pl
}

\begin{abstract}
The study presented in this paper is focused on the analysis of biomechanical effects of several impact forces exerted on the orbital skeleton. Three different cases of force application reflecting three sport-related situations have been investigated. Time histories of the impact force as well as area of its application have been considered. As a tool to carry out numerical simulation, ANSYS Workbench software platform has been used. The analysis showed that the most dangerous is the force applied to the lower part of orbital margin. In this case, the entire zygomatic bone may be damaged due to strong bending load.
\end{abstract}

Keywords: sports-related injuries, orbital bones, tennis ball impact, finite element method (FEM)

\section{Introduction}

There are some kinds of sports (boxing, full contact martial arts) where, by definition, athletes purposely hit or collide with each other or with inanimate objects (including the ground) with a great force. There are also many sports, where the rules do not provide for a direct collision with an opponent or stationary and nonstationary objects, but in practice such events occur quite often. An example of this kind of sports can be basketball, handball, tennis or golf. Paradoxically, in this latter group of sports, due to the unexpected nature of the impact and the resulting lack of prepared player reaction, the consequences can be even more serious.

Among different types of sport injuries, one of the most frequent, and the most dangerous at the same time, are head injuries (Bullock et al., 1999; Rhee et al., 2002; Rubin and Winograd, 2002; Echlin et al., 2005; Gomes et al., 2006; Ceallaigh et al., 2007; Siswanto and Hua, 2012). The consequences of these injuries can be very hazardous to health and even life of competitors, because the head is a place where vitally important organs, like brain or organs of sight, hearing and balance, are located.

In the literature, one can find many reports on sport accident statistics as well as description of sport-related injuries, their causes and ways to treat their effects. For example, Mourouzis and Koumura (2005) assessed a spectrum of maxillofacial fractures sustained during sports in Greece, discussed the aetiology in different sports and suggested protective measures. The evaluated factors were: type of sport involved, age, sex, mechanism of injury, site of fractures, associated nonmaxillofacial injuries and mode of treatment. According to the authors' findings, facial fractures during sports mainly affect young people and the majority of patients are amateur athletes. Team sports are responsible for frequent occurrence of facial fractures. A retrospective study on facial fractures was carried out by Klenk and Kovacs (2003). The study included 144 patients with the mean age of 26.5 years; the most frequently injured patients belonged to the 16- up to 20 -year-old group. A total of $53.4 \%$ of the patients suffered isolated mandibular fractures, $32.6 \%$ 
had isolated midface fractures, and $13.8 \%$ had combined midface and mandibular fractures. A prospective study involving patients presenting facial fractures sustained during sports was carried out by Maladière et al. (2001). The majority of injuries involved the mandible $(34.4 \%)$, zygomatic bone (23.4\%) and the nasal bone (15.6\%). The sporting activities were classified as either contact or noncontact sports. Gomes et al. (2006) presented a 5-year retrospective study of zygomatico-orbital complex and zygomatic arch fractures. The majority of fractures were sustained by males between 21 and 40 years old, usually resulting from falls, altercations and bicycle accidents. Unilateral fractures of zygomatico-orbital complex were present in 266 patients $(71.69 \%)$, bilateral fractures were found in 22 cases $(5.93 \%)$ and unilateral zygomatic arch fractures occurred in 39 cases (10.51\%).

This brief literature review shows how serious problems may be associated with sport-related harms in the orbital region, and how important is biomechanical analysis of their causes and consequences. The results of investigations may help one to determine the ways to avoid or minimize the effect of injury or indicate possible repair options in the case of bone damage. In this paper, some examples of possible blows in the vicinity of eye socket, and the resulting mechanical upshots, are considered.

\section{Method and model}

Due to complex geometrical and material structure of the facial bones, the only effective and thus commonly used numerical tool for conducting strength tests in this type of objects is the finite element method (FEM) (Voo et al., 1996; Sahoo et al., 2015; Tse et al., 2017). Assuming that the prepared FEM model reflects the real object appropriately, this method allows for understanding and correct assessment of the mechanism of fracture formation in this part of the skull (Takizawa and Takahashi, 1995; Al-Sukhun et al., 2006; Asgharpour et al., 2014; Bontrager and Lampignano, 2014; Huempfner et al., 2014).

Usually, while preparing a FEM model, one has to reconcile two conflicting requirements: ensure conformity of the model with the real object (what is generally associated with a large number of elements), and on the other hand, reduce time of calculation. In the present study, in order to meet the above demands, the reproduced area of the skull was limited to a minimum, i.e. to the right orbit excluding the nasal bone and nasal septum (Fig. 1).

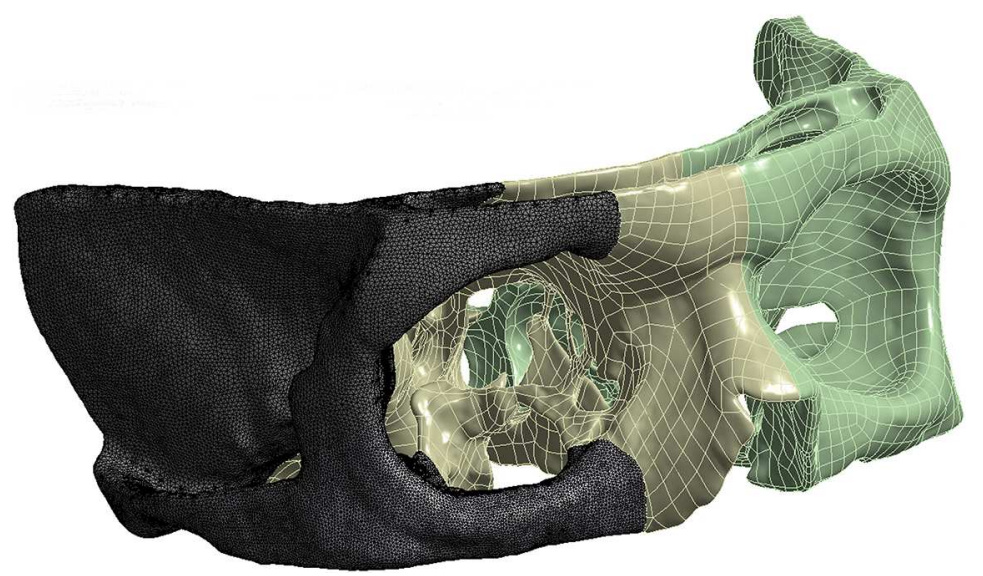

Fig. 1. Part of a skull subjected to FEM analysis

A geometric model of the aforementioned orbit part was obtained based on the skull CT (Computed Tomography) scans of a healthy 35 year old man by converting DICOM files into a .sat format supported by ANSYS program. The model contained only the bone structures 
without soft tissue. This simplification was justified by the fact that in the surveyed craniofacial area, soft tissue is practically limited to the skin shell and minimally affect the values of the resulting impact strain and stress, and does not affect their distribution. Such a model was analysed in an environment of ANSYS Workbench platform named Transient numerical.

Before taking the main examinations, a series of tests to select the best model mesh has been performed. Several models with a different number of nodes and elements, as well various shapes and sizes of the elements, have been estimated. Finally, it was decided to use a model built from 771716 nodes and 845591 tetrahedron elements. This type of spatial elements of dimensions from $0.5 \mathrm{~mm}$ up to $0.7 \mathrm{~mm}$ ensured $99.7 \%$ compliance with the grid quality criterion and good representation of the real object (Fig. 2).

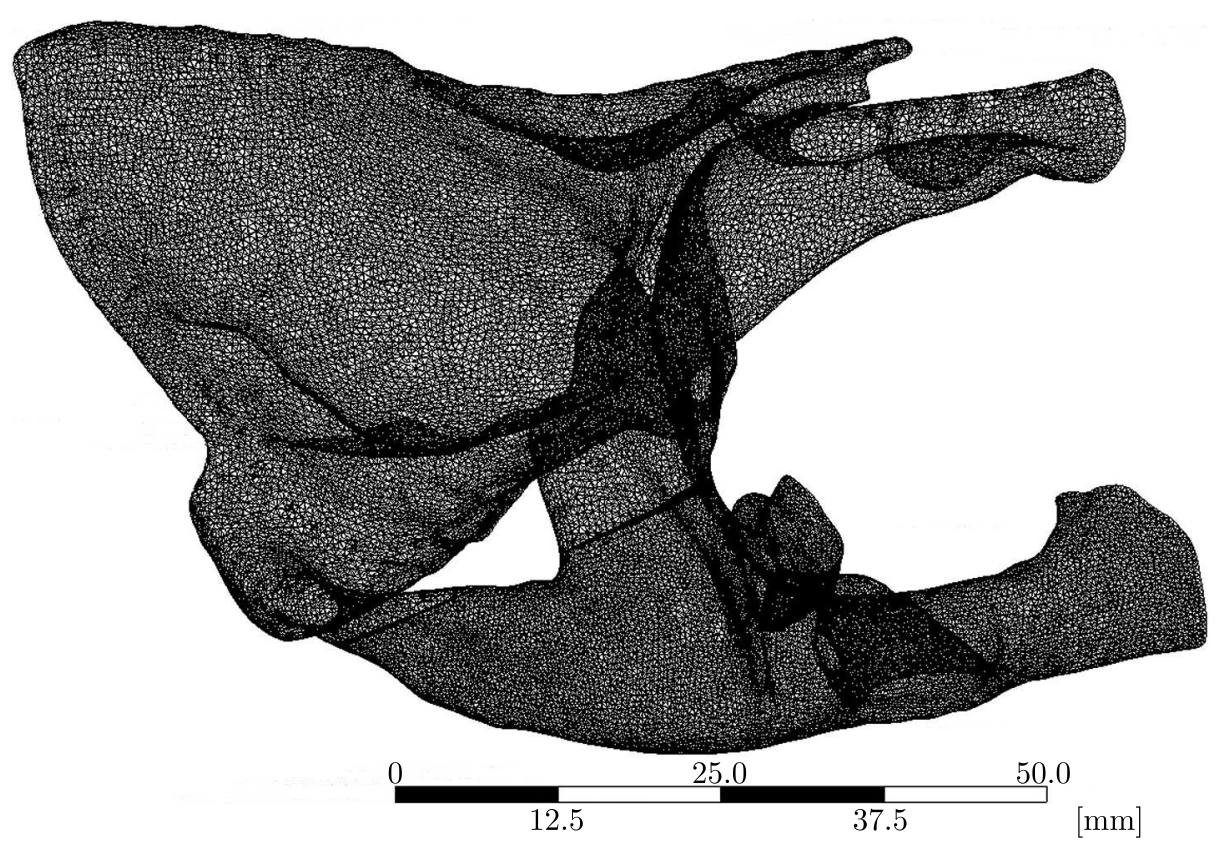

Fig. 2. Mesh of the orbital model

Boundary conditions reflecting the influence of the adjacent facial bones on the fragment of cranium under consideration were defined using two types of fastening (Fig. 3). First of them, named Fixed support, deletes six degrees of freedom at each node (in the nasal bone and in the nasal septum). The second one, named Displacement, precludes all translational displacements at each node (deletes the translational degrees of freedom directed along the $x, y$ and $z$ axes). This kind of fastening has been applied in the junction of the model with frontal, parietal and temporal bones.

The properties of the bone material were assumed based on data taken from the specialist literature: Young's modulus of elasticity $E=12200 \mathrm{MPa}$, Poisson's ratio $\nu=0.21$ and density $\rho=1.8 \mathrm{~kg} / \mathrm{m}^{3}$ (Diaw et al., 1997). The above-defined material parameters were adopted as average values for the whole structure.

During the calculation, geometrical nonlinearity has been taken into account. Since the numerical simulations were of a qualitative nature, the influence of distribution of soft tissues around the facial skeleton was ignored. In order to determine the probable place of bone damage, a bilinear material was introduced, what restricted the range of the material curve to the elastic area. 


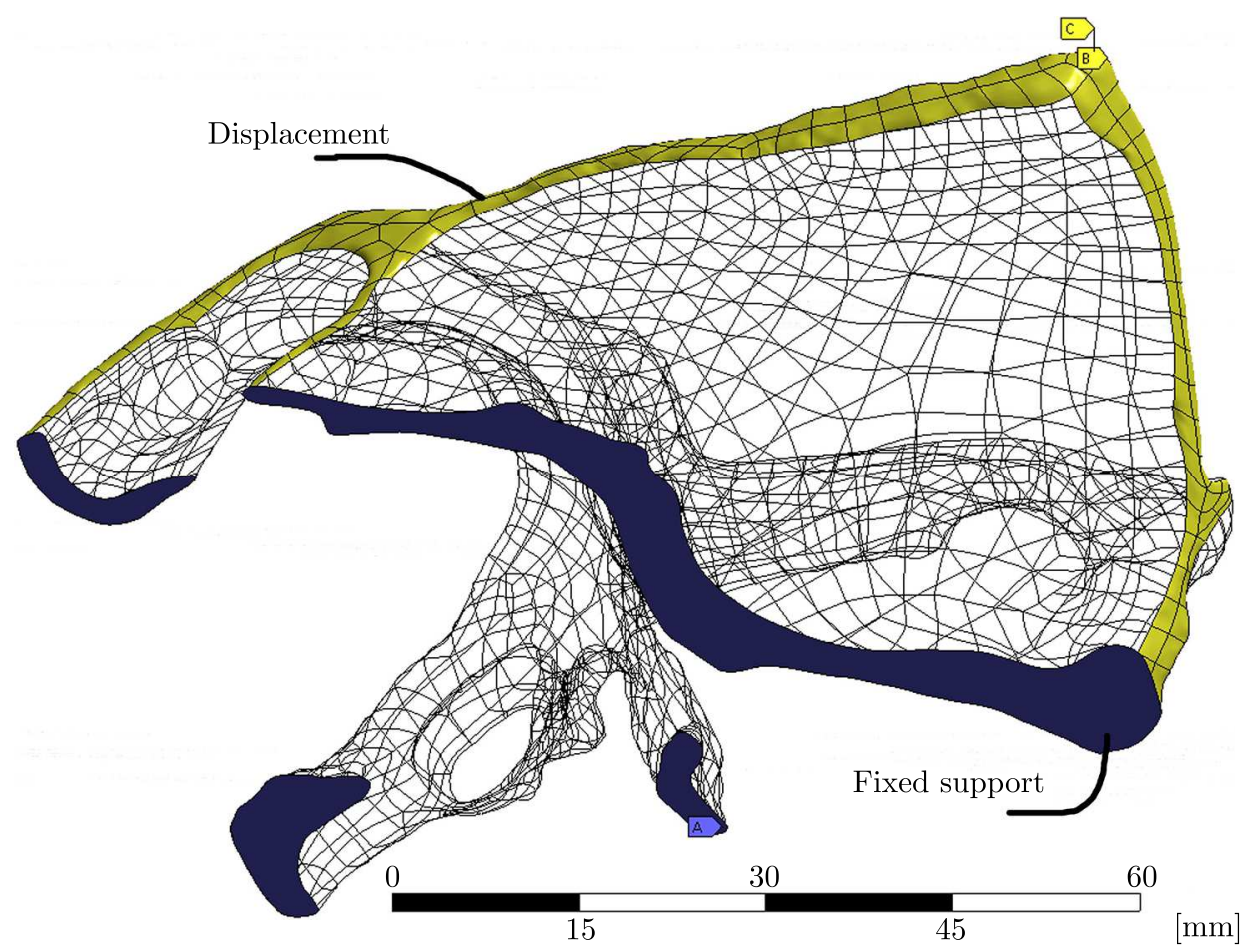

Fig. 3. Location of two types of boundary conditions: Displacement and Fixed support

\section{Results}

Three cases reflecting the mechanical response of the orbital bones to three different impact forces generated during sport activities have been analysed. Such forces may result from golf/tennis ball hitting, fist punch or elbow strike, for example.

\subsection{Fixed impact force analysis}

In the first stage of the study, in order to simplify the analysis, a fixed force value equal to $275 \mathrm{~N}$ as well as a constant case-dependent force application area have been assumed. The investigated cases differed in location and area of application of the impact force, and were defined as follows: (a) force applied to the lower orbital margin, (b) force applied to the zygomatic process of the frontal bone, (c) force applied to the orbital rim (Fig. 4).

As a result of FEM analysis, a series of reduced von Mises stresses and displacement maps has been obtained. The reduced stress distribution for three analysed cases is shown in Fig. 5.

(a)

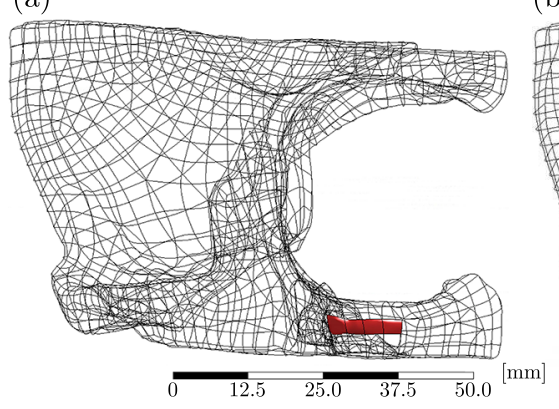

(b)

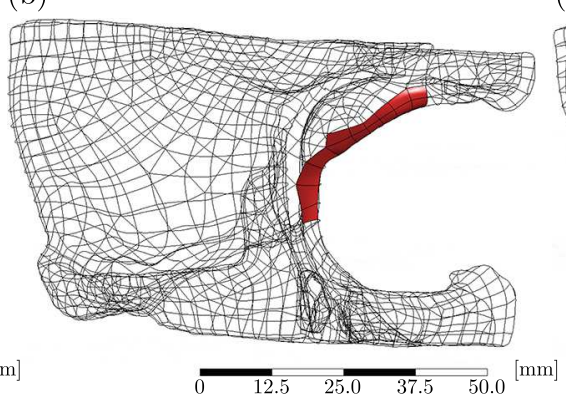

(c)

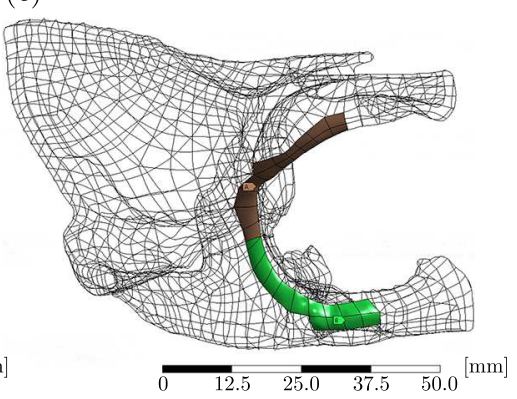

Fig. 4. Three cases of force application: (a) force applied to the lower orbital margin, (b) force applied to the zygomatic process of the frontal bone, (c) force applied to the orbital rim 
(a)
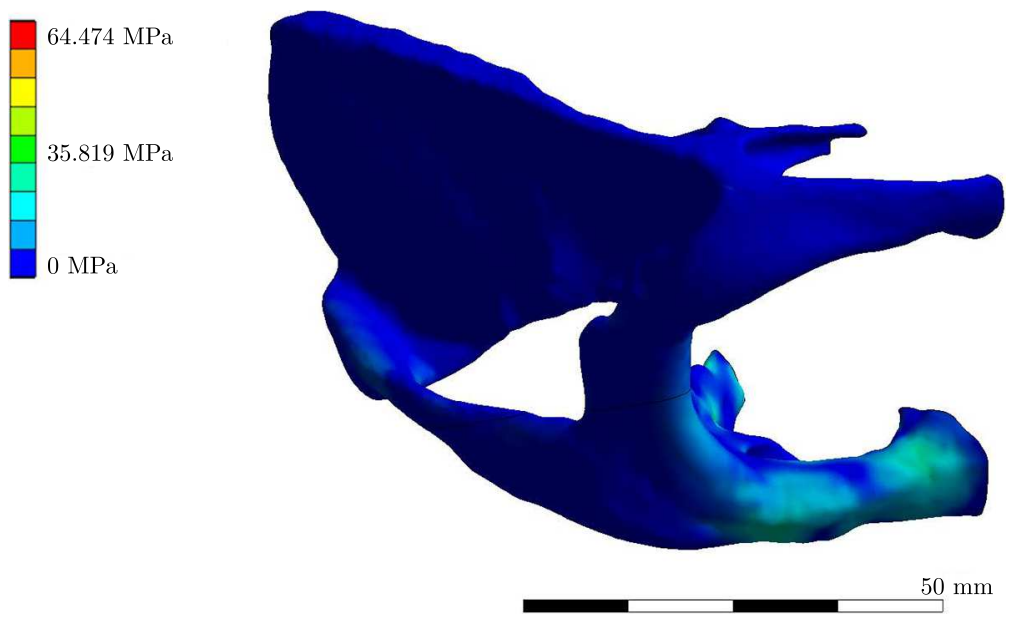

(b)

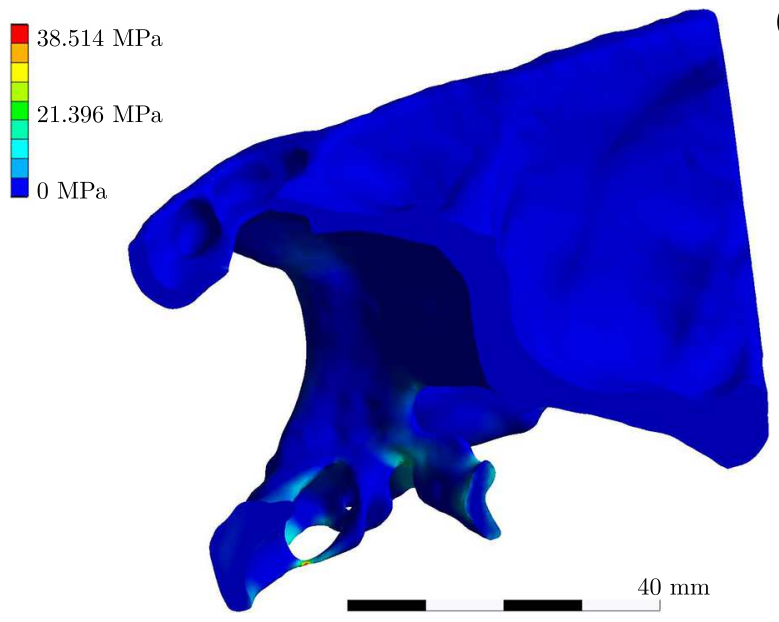

(c)

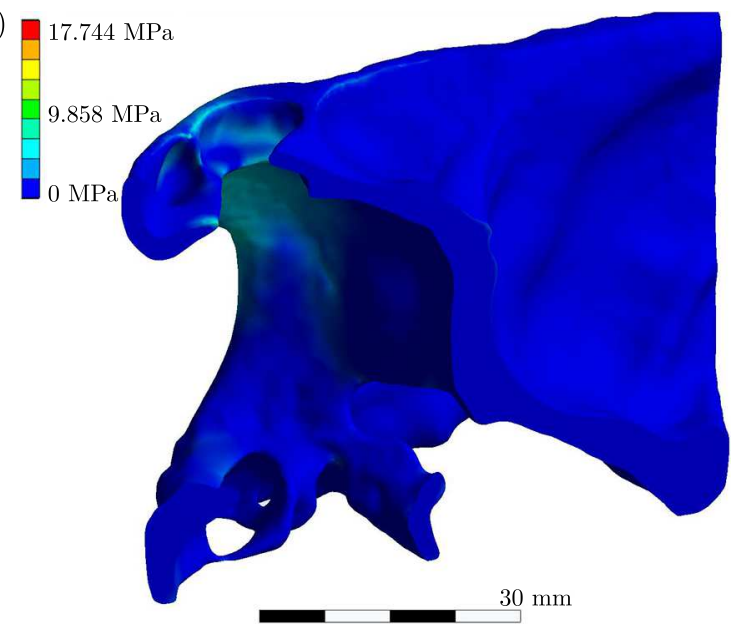

Fig. 5. Maps of reduced von Mises stress: (a) force applied to the lower orbital margin, (b) force applied to the zygomatic process of the frontal bone, (c) force applied to the orbital rim

According to the calculations, the most unfavourable load is a force applied to the lower margin (case a). For this type of blow, the weakest region is the lower part of the zygomatic bone (Fig. 5a). In this case, the load is transferred from the front part of the eye socket on its rear part, which causes enlargement of the damage threatened zone.

Similar conclusions can be also reached by analysing displacements resulting from the same force application (Fig. 6). In this case, the maximum displacement is concentrated in the lower part of the zygomatic bone and descends into the orbit and zygomatic arch, which acts as a specific bumper absorbing a significant part of impact energy.

\subsection{Analysis of impact effects when force magnitude and contact area are time-dependent}

Knowing that the most dangerous situation takes place when the force is applied to a small area of the inferior margin, a deepened analysis of this case, taking into account the change in time of both the force magnitude and the surface area to which it is applied, has been carried out. This case corresponds to the tennis ball hitting the lower part of the eye socket.

The investigations were based on the results of experimental studies on tennis balls dynamic properties described in Cross (1999). From a variety of cases analyzed in the aforementioned paper, the outcomes concerning Wilson US Open ball with a mass of $56.5 \mathrm{~g}$ and a diameter of $65 \mathrm{~mm}$, at an impact speed of $13.5 \mathrm{~m} / \mathrm{s}$, have been used in numerical simulations. Following these data, the real pressure of a tennis ball exerted on the lower orbital margin was presented as a 


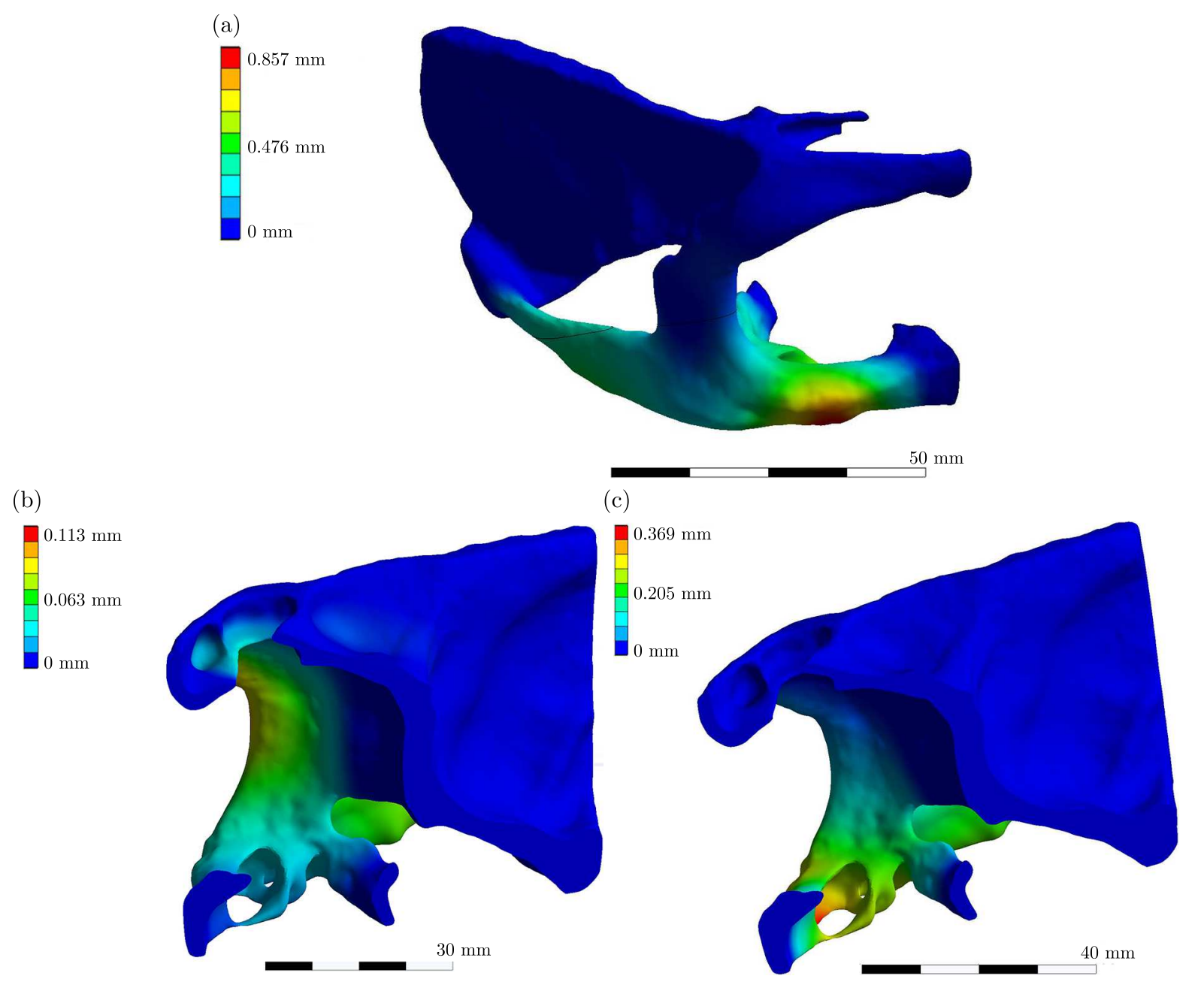

Fig. 6. Maps of displacement: (a) force applied to the lower orbital margin, (b) force applied to the zygomatic process of the frontal bone, (c) force applied to the orbital rim

superposition of two force components with different amplitudes and different time histories. Such an approach reflects dissimilar properties of the cloth covering of the ball and its rubber wall, observed during compression. The total force resulting from these two components, taking into account changes in the contact area of the ball with the eye socket, was determined for three time instants during the impact. Its values were respectively equal to: $275 \mathrm{~N}$ for $t=2 \mathrm{E}-4 \mathrm{~s}$, $400 \mathrm{~N}$ for $t=6 \mathrm{E}-4 \mathrm{~s}$ and $575 \mathrm{~N}$ for $t=1.8 \mathrm{E}-3 \mathrm{~s}$. On this basis, an approximate graph of the resulting force was created (see Fig. 7). In the analysis, only a part of the force diagram from the beginning of the impact until the maximum value reached at $1.8 \mathrm{~ms}$ has been considered. The rest of the chart is not significant from the point of view of strength analysis.

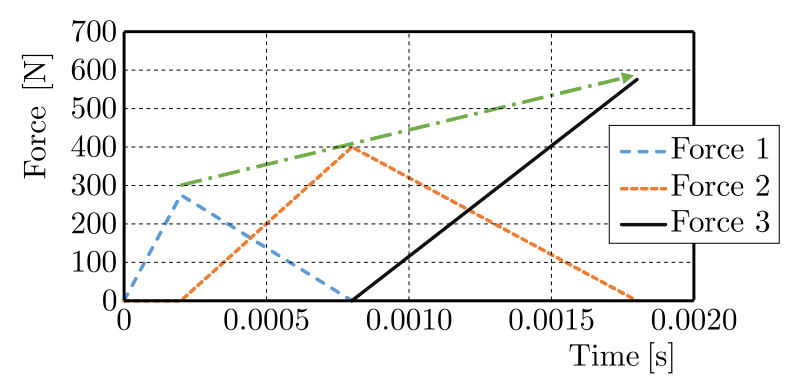

Fig. 7. Composition and time history of the impact force 
As mentioned earlier, the contact area between a tennis ball and orbital bones during the impact has been treated as time-dependent. A simplified way to determine the magnitude of the contact area is presented in Fig. 8. Knowing the radius $R$ of the ball and its deflection value $y$, one can specify the parameter $a$ that approximately defines the area of contact.

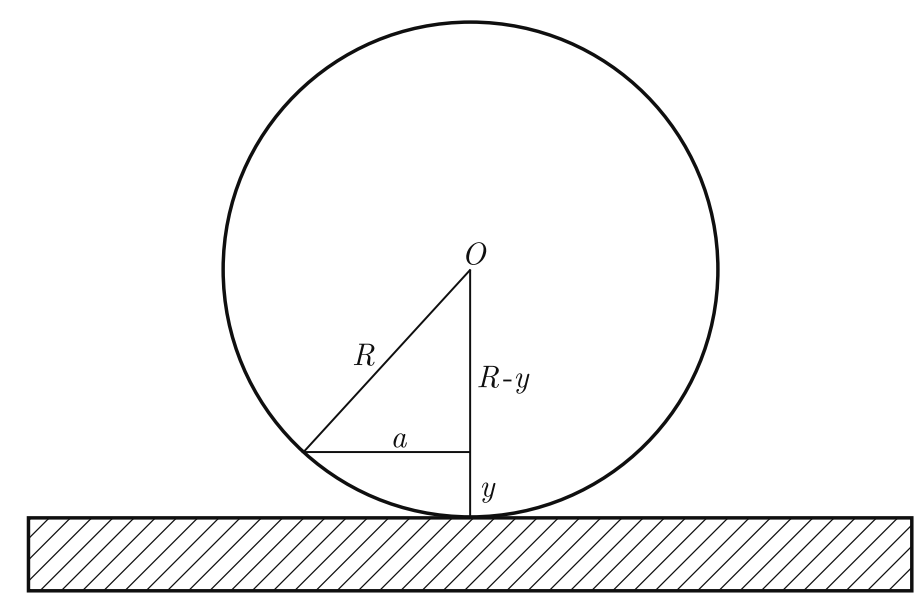

Fig. 8. Scheme of assessment of the contact area between a tennis ball and the eye socket ( $R$ - ball radius, $y$ - ball deflection, $a$ - radius of the outer circle of ball-eye socket contact)

(a)

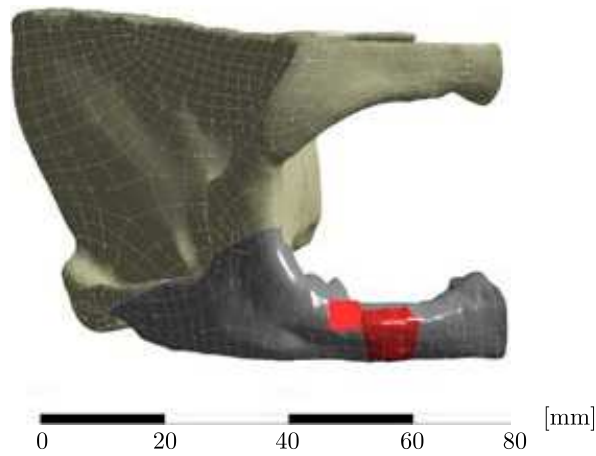

(b)

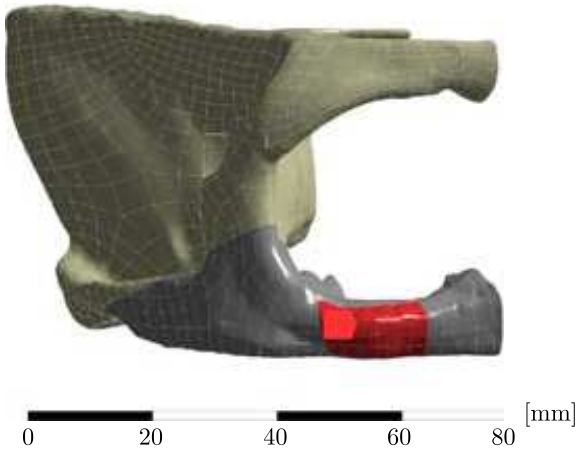

(c)

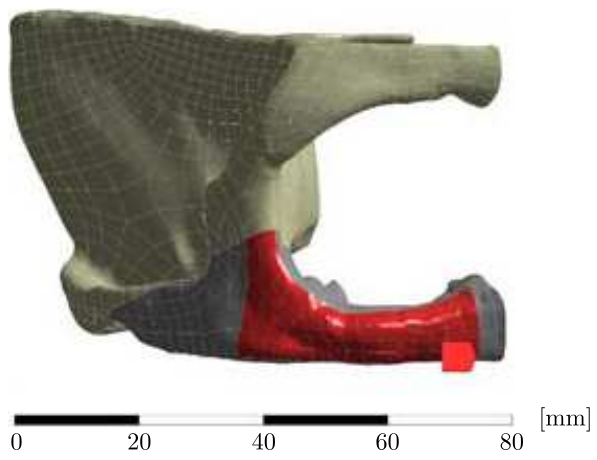

Fig. 9. Estimated surfaces of force application in different phases of the impact: (a) $t=2 \mathrm{E}-4 \mathrm{~s}$, (b) $t=6 \mathrm{E}-4 \mathrm{~s},(\mathrm{c}) t=1.8 \mathrm{E}-3 \mathrm{~s}$

Estimated contact surfaces between the tennis ball and the lower part of orbital rim for three different moments during the impact are shown in Fig. 9. In the next stage of computation and analysis of possible areas of bone damage resulting from variable load over time, the boundary conditions were optimized. Supports stiffening the system, which in the first stage of calculations replaced the "cut off" bone fragments, were replaced with supports with adjustable stiffness (Fig. 10). As a result of many analyzes and possible configurations of the boundary conditions, the rigidity of the supports was selected in such a way as to obtain, in the end, a similar 
image of bone destruction comparable to the real image corresponding to a zygomaticomaxillary complex fracture (tripod fracture) (see Fig. 11). The maps of reduced stress (Fig. 12) illustrate the potential place of destruction of bone fragments (red) as well as the way of load dissipation in the examined skull fragment (Fig. 13). Observe that supports are involved in load transfer which corresponds to energy absorption.

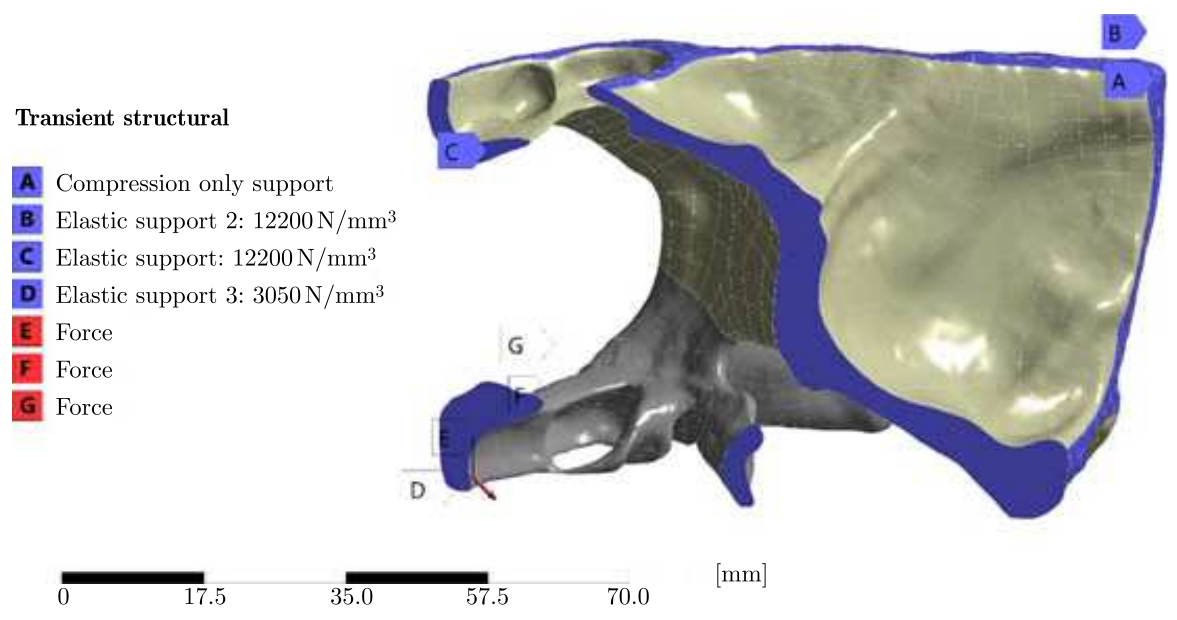

Fig. 10. Boundary conditions: assumed supports and their stiffness corresponding to bone properties

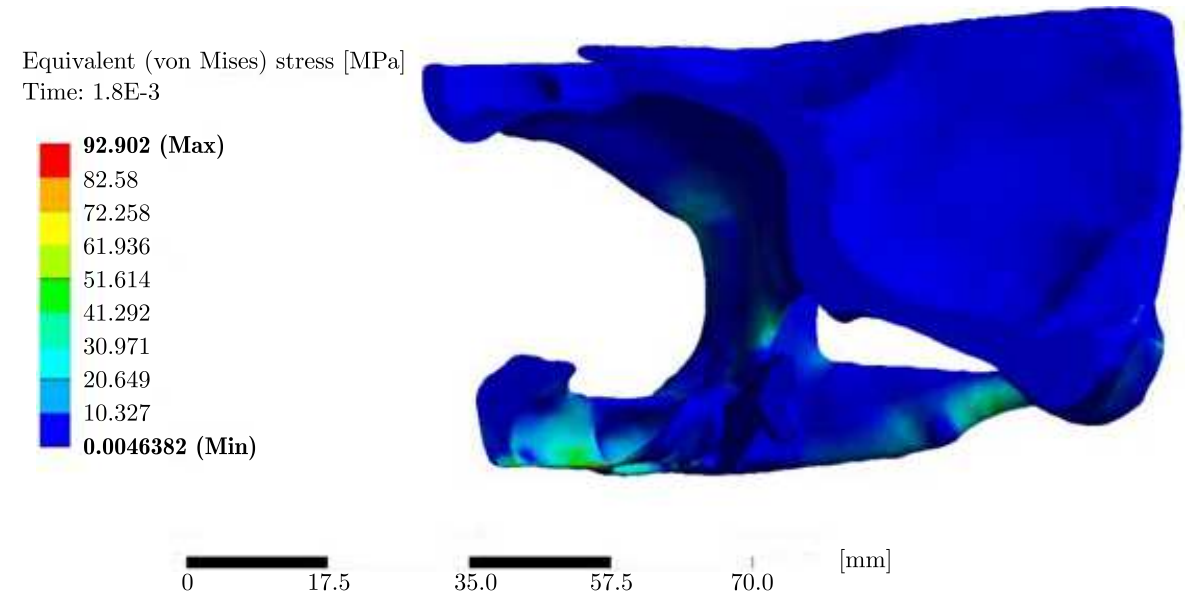

Fig. 11. Map of reduced stresses in the final load phase $(t=1.8 \mathrm{E}-3 \mathrm{~s})$
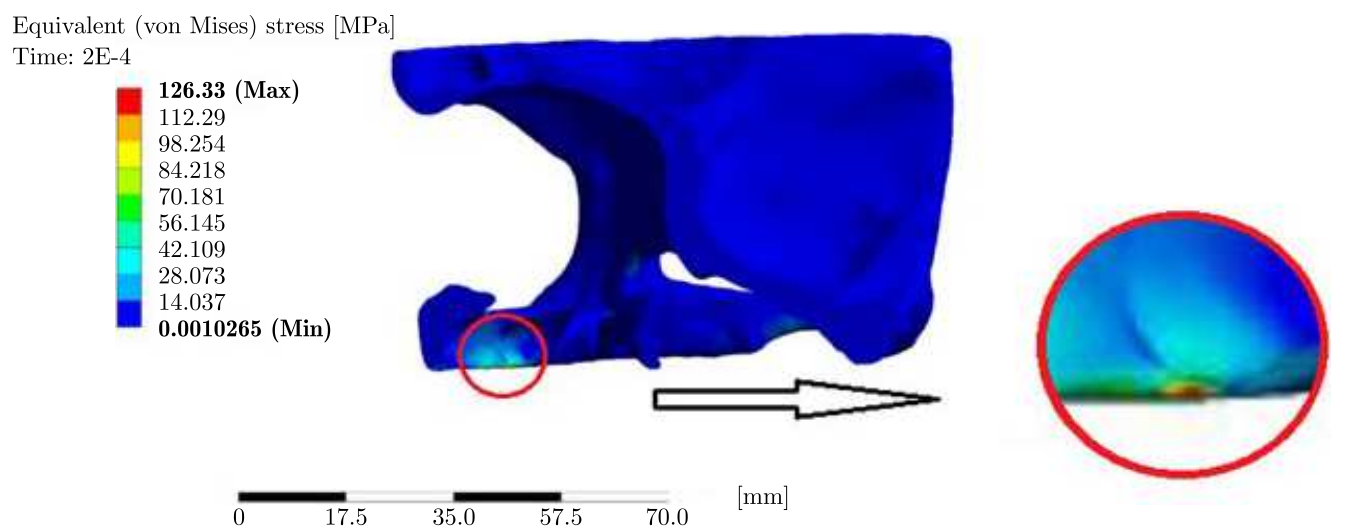

Fig. 12. Map of reduced stresses at $t=2.0 \mathrm{E}-4 \mathrm{~s}$ (the maximum effort area is marked in red) 


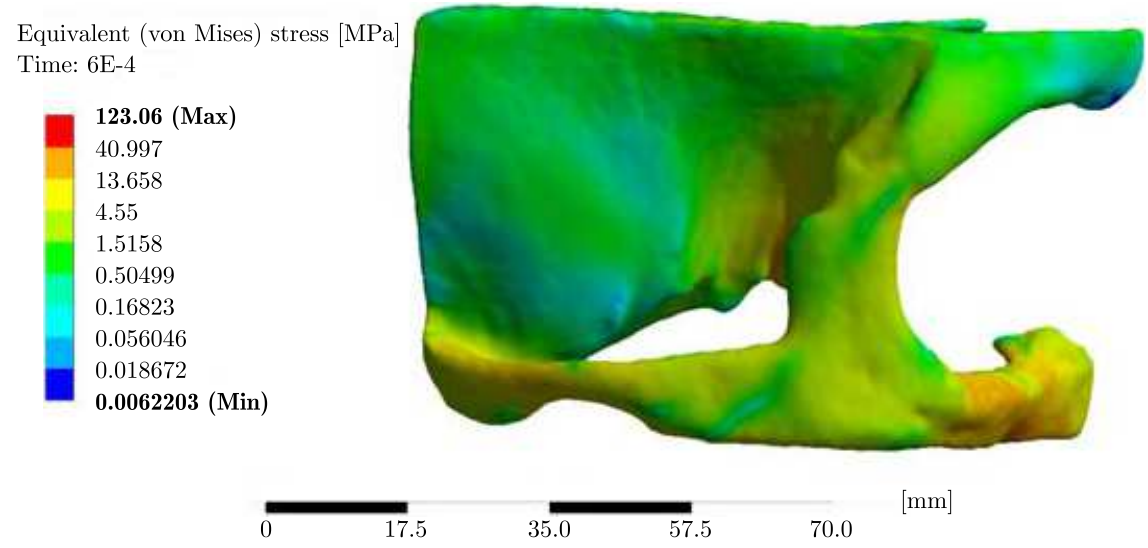

Fig. 13. Map of reduced stresses at $t=6.0 \mathrm{E}-4 \mathrm{~s}$ with areas of the maximum effort and dissipation

The results of step-by-step stress and displacement computation for evenly distributed time values from 0 to $1.8 \mathrm{~ms}$ are presented in Fig. 14.
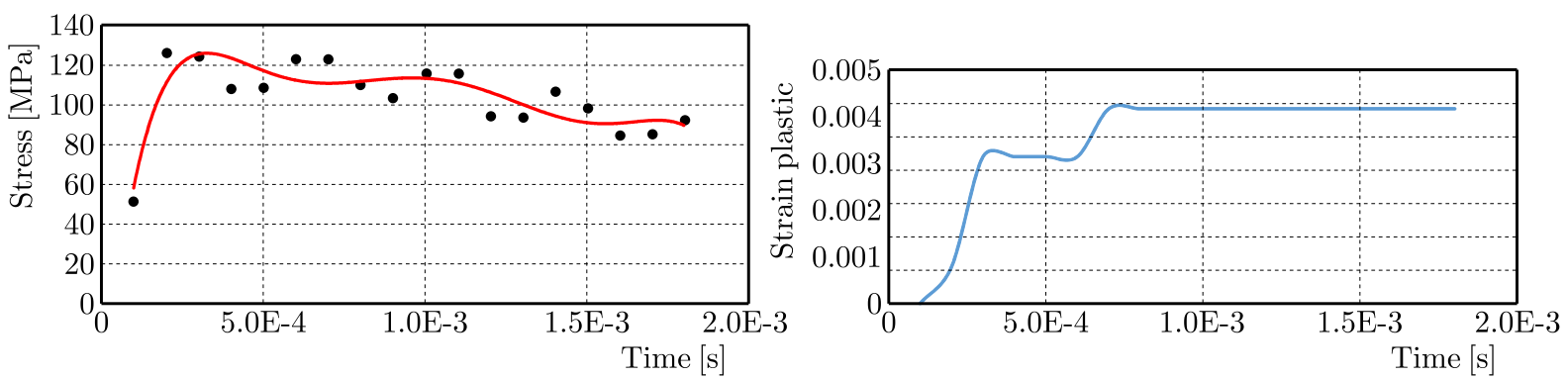

Fig. 14. Variability of von Mises stress and strain plastic deformation during the impact

The analysis shows that the fracture could occur in the area, where the rear part of the zygomatic arch connects with the temporal bone. It should be noted that the most dangerous is the time instant before the force reaches its maximum value. This means that a break would occur in a time between $2 \mathrm{E}-4 \mathrm{~s}$ and $7 \mathrm{E}-4 \mathrm{~s}$, as illustrated in Fig. 14. This figure shows von Mises reduced stress and plastic deformation values as criteria for bone destruction. Each of these quantities reaches its maximum value in a similar time, i.e. clearly earlier than the maximum force is achieved. The reason for this is that the contact area between the ball and the bone grows faster than the impact force, thereby reducing the stress value.

In the framework of the research, much attention was paid to the interpretation of the results concerning the analysis of the probable place of fracture. The assessment criterion was established on the basis of the maps of equivalent stress. It is known that in real situations plastic deformation in the bone tissue does not occur. Therefore, this observation was used to assess possible damage spots. In the analyzed case, the place of potential bone injury is localized in the posterior part of the zygomatic bone (Fig. 15). A bone fracture in this region can result in damage of the entire orbital cone. The consequence would be dislocation of the eyeball, manifesting in the difficulty in eye moving and diplopia.

In addition, comparing the map of the reduced stress with the displacement map, one can notice that the locations of their maximum values coincide. The maximum deflection of the zygomatic arch is found in its central part, and is approximately equal to $0.7 \mathrm{~mm}$. This demonstrates relatively high elasticity of this region of the orbital cavity. 


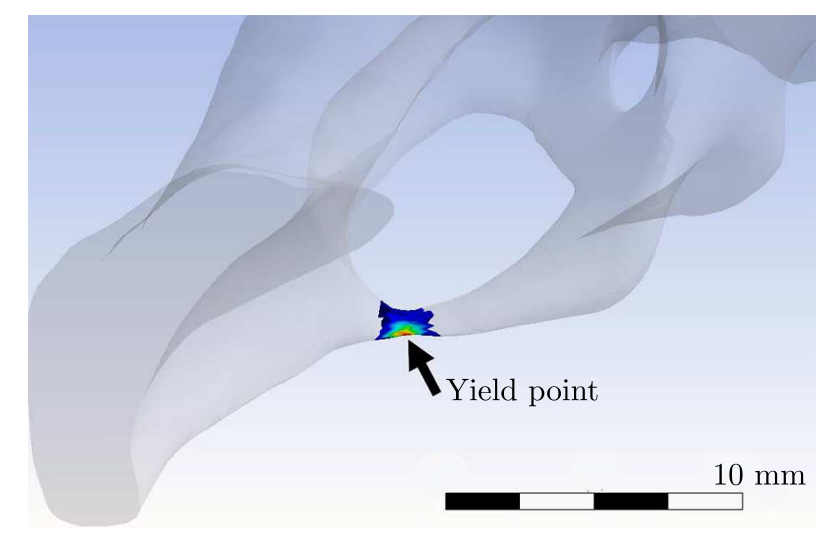

Fig. 15. Yield point location - probable place of fracture

\section{Concluding remarks}

Three cases concerning different impact forces acting on the eye socket have been analyzed. All of them reflect typical situations which can occur during sports practicing. The first force was applied to a small part of the lower orbital margin, the second to the zygomatic process of the frontal bone, and the third one to the orbital rim. For each case, a numerical simulation using a simplified time-independent loading model and ANSYS Workbench platform was carried out. The results clearly indicate that the first case is the most unfavorable from the point of view of bone strength. The two other cases do not pose a serious threat to the eye socket. This statement is in accordance with medical findings and results from the strong bone structure in these orbital regions and a relatively large area of force application.

In the most disadvantageous case, i.e. when the force is applied to the lower part of orbital margin, the posterior part of the zygomatic bone, which can be defined as strengthening (supporting) element of the orbit, would be fractured. As a consequence of such an incident, the entire lower zygomatic bone would be damaged due to the strong bending load. Furthermore, this damage would propagate to the rear part of the eye socket, causing a loss of support for the eyeball.

In order to check whether the simplified loading model is correct and gives satisfactory results, detailed verification studies, more precisely reflecting the real impact process, have been carried out. For this purpose, a quasi-dynamic load model taking into account the change in time of both the force magnitude as well as its area of application has been considered. This model corresponds to a tennis ball hitting the lower edge of the eye socket. The results, on a qualitative level, correspond with the previously obtained outcomes for the simple load model. Numerical simulation demonstrated that the location of the yield point coincides with its placement found during the earlier analysis. Moreover, the designated location matches the place of probable fracture indicated by the reduced stress and displacement maps obtained formerly by using a simplified load model.

These observations show that for the correct assessment of the orbital bones effort during the impact, the use of a simplified loading model is sufficient and leads to time-saving calculations.

In addition, it was found that the moment at which orbital bone fracture (yield point) may occur does not coincide with the time at which the force achieves its maximum value. This is due to the fact that the rate of the force value increase is not the same as the rate of growth in its area of application. 


\section{References}

1. Al-Sukhun J., Lindqvist C., Kontio R., 2006, Modelling of orbital deformation using finite-element analysis, Journal of the Royal Society Interface, 3, 7, 255-262

2. Asgharpour Z., Baumgartner D., Willinger R., Graw M., Peldschus S., 2014, The validation and application of a finite element human head model for frontal skull fracture analysis, Journal of the Mechanical Behavior of Biomedical, 33, 16-23

3. Bontrager K.L., Lampignano J.P., 2014, Textbook of Radiographic Positioning and Related Anatomy, Elsevier, Mosby

4. Bullock J.D., Warwar R.E., Ballal D.R., 1999, Mechanisms of orbital floor fractures: a clinical, experimental, and theoretical study, Transactions of the American Ophthalmological Society, 97, $87-113$

5. Ceallaigh P.O., Ekanaykaee K., Beirne C.J., Patton D.W., 2007, Diagnosis and management of common maxillofacial injuries in the emergency department. Part 3: Orbitozygomatic complex and zygomatic arch fractures, Emergency Medicine Journal, 24, 2, 120-122

6. Cross R., 1999, Dynamic properties of tennis balls, Sports Engineering, 2, 23-33

7. Diaw B.M., Willinge R., Kang H.-S., 1997, Finite elements modelling of bone material discontinuity in case of skull fracture, Proceedings of the 1997 International Conference on the Biomechanics of Impact, Hannover, Germany

8. Echlin J.S., Upshur R.E., Peck D.M., Skopelua E.N., 2005, Craniomaxillofacial injury in sport: a review of prevention research, British Journal of Plastic Surgery, 39,5, 254-263

9. Gomes P.P., Passeri L.A., Barbosa J.R., 2006, A 5-year retrospective study of zygomaticoorbital complex and zygomatic arch fractures in Sao Paulo State, Brazil, Journal of Oral and Maxillofacial Surgery, 64, 1, 63-67

10. Huempfner-Hierl H., Schaller A., Hierl T., 2014, Biomechanical investigation of the supraorbital arch - a transient FEA study on the impact of physical blows, Head and Face Medicine, 10, $13-17$

11. Klenk G., Kovacs A., 2003, Etiology and patterns of facial fractures in the United Arab Emirates, Journal of Craniofacial Surgery, 14, 1, 78-84

12. Lipa S., Mrozowski J., Awrejcewicz J., 2015, Analysis of orbital strain and stress caused by multidirectional forces generated during a ball impact, [In:] Dynamical Systems: Mechatronics and Life Sciences, J. Awrejcewicz, M. Kaźmierczak, J. Mrozowski, P. Olejnik (Eds.), TU of Lodz, 295-304

13. Maladière E., Bado F., Meningaud J.-P., Guilbert F., Bertrand J.C., 2001, Aetiology and incidence of facial fractures sustained during sports: a prospective study of 140 patients, International Journal of Oral and Maxillofacial Surgery, 30, 4, 291-295

14. Mourouzis C., Koumoura F., 2005, Sports-related maxillofacial fractures: a retrospective study of 125 patients, International Journal of Oral and Maxillofacial Surgery, 34, 635-638

15. Rhee J.S., Kilde J., Yoganadan N., Pintar F., 2002, Orbital blowout fractures: experimental evidence for the pure hydraulic theory, Archives of Facial Plastic Surgery, 4, 2, 98-101

16. Rubin M.L., Winograd L.A., 2002, Taking Care of Your Eyes, Triad Publishing Company

17. Sahoo D., Deck C., Yoganandan N., Willinger R., 2015, Influence of head mass on temporo-parietal skull impact using finite element modeling, Medical and Biological Engineering and Computing, 53, 9, 869-878

18. Siswanto W.A., Hua C.S., 2012, Strength analysis of human skull on high speed impact, International Review of Mechanical Engineering, 6, 7, 1508-1514

19. TAkizawa Y., Takahashi K., 1995, Three-dimensional finite element analysis of blowout fractures, Nippon Ganka Gakkai Zasshi, 99, 8, 972-979 
20. Tse K.M., TAn L.B., YAng B., TAn V.B., Lee H.P., 2017, Effect of helmet liner systems and impact directions on severity of head injuries sustained in ballistic impacts: a finite element (FE) study, Medical and Biological Engineering and Computing, 55, 4, 641-662

21. Vinger P.F., Duma S.M., Crandall J., 1999, Baseball hardness as a risk factor for eye injuries, Archives of Ophthalmology, 117, 3, 354-358

22. Voo L., Kumaresan S., Pintar F.A., Yoganandan N., Sances A. Jr., 1996, Finite-element models of the human head, Medical and Biological Engineering and Computing, 34, 5, 375-381

Manuscript received November 27, 2018; accepted for print December 18, 2019 